rev.relac.int.estrateg.segur.10(2):43-68,2015

\title{
LA TOMA DE REHENES COMO ACTO TERRORISTA INTERNACIONAL. ANÁLISIS DE LA OPERACIÓN DE RESCATE "CHAVÍN DE HUANTAR"
}

\author{
Ariel Álvarez Rubio**
}

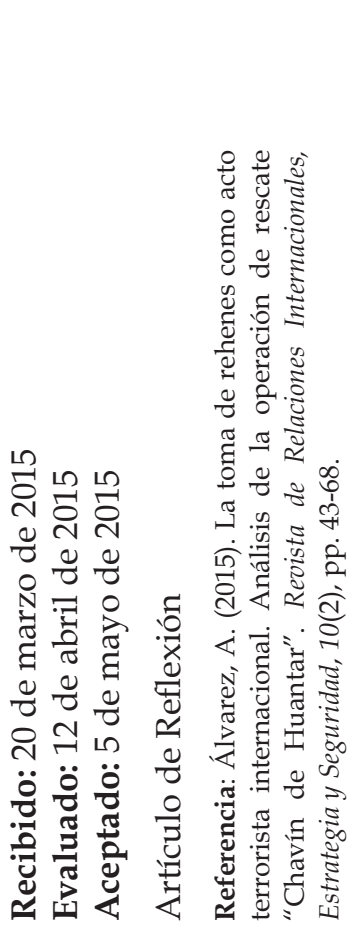

\section{RESUMEN}

La toma de rehenes es un fenómeno que afecta a muchos países, y donde se refleja la cambiante relación existente entre la política y la guerra contra el terrorismo. Para combatir esta amenaza, los gobiernos se ven obligados a actuar con todas las capacidades disponibles y en ocasiones, los grandes principios y los escrúpulos morales son obviados por los agentes del Estado encargados de la lucha contraterrorista. Por estos motivos, el presente artículo analiza la operación de rescate de rehenes "Chavín de Huantar", destinada a liberar a 72 personas secuestradas por el Movimiento Revolucionario "Tupac Amaru" (MRTA). El objetivo del estudio es adentrarse en el complejo 'dilema' referido a si en una 'democracia liberal' se puede considerar aceptable 'combatir el terror con una violencia sin límites' y si en esta época de terrorismo,

Artículo de reflexión, resultado de los avances de investigación de la tesis doctoral: La toma de rehenes como acto terrorista internacional: Un análisis integrado de los casos de Perú y Colombia, del programa de Doctorado en Estudios Americanos, mención Estudios Internacionales, de la Universidad Santiago de Chile (USACH).

** General de la Brigada Aérea (Ret.) de la Fuerza Aérea de Chile. Ingeniero Sistemas de Armas. Magíster en Ciencias Militares y Doctor en Estudios Americanos. Correo electrónico: aalvarez@fach.mil.cl 
podemos resistirnos a la tentación de 'no tener piedad'. A nivel metodológico, se realiza una exhaustiva revisión bibliográfica y un estudio de campo cuyos resultados permiten comprobar que no se puede otorgar concesiones a los terroristas en materia de toma de rehenes, pero si se debe priorizar el empleo de los recursos más apropiados para que los ciudadanos víctimas de este flagelo salgan ilesos de esta condición.

Palabras clave: Lucha Antiterrorista, Rescate de Rehenes, Operaciones Especiales, Terrorismo.

\title{
HOSTAGE TAKING AS AN INTERNATIONAL TERRORIST ACT: ANALYSIS OF THE RESCUE OPERATION "CHAVÍN DE HUANTAR"
}

\begin{abstract}
Hostage taking is a phenomenon that affects many countries, where the changing relationship between politics and the war against terrorism is reflected. To combat this threat, governments are forced to act with all available capacities and sometimes major principles and moral scruples are ignored by government agents in charge of the fight against terrorism. This article analyzes the hostage rescue operation "Chavin de Huantar" aimed at freeing 72 people kidnapped by the "Tupac Amaru" Revolutionary Movement (MRTA). The aim of the study is to explore the 'dilemma' complex issue of whether a 'liberal democracy' can be considered acceptable 'fight terror with unlimited violence' and if in this age of terrorism, we can resist the temptation to 'have no mercy'. As a methodology level, a comprehensive literature review and field study whose results allow verifying that it cannot grant concessions to terrorists in hostage is done, but whether to prioritize the use of the most appropriate resources for citizens' victims of this scourge come out unscathed from this condition.
\end{abstract}

Keywords: Counter Terrorism, Hostage Rescue, Special Operations, Terrorism.

\section{TOMADA DE REFÉNS COMO ATO TERRORISTA INTERNACIONAL: ANALISE DA OPERAÇÃO DE RESGATE "CHAVÍN DE HUANTAR"}

\section{RESUMO}

Tomada de reféns é um fenômeno que afeta muitos países, onde a evolução das relações entre a política ea guerra contra o terrorismo é refletida. Para combater essa ameaça, os governos são obrigados a agir com todas as capacidades disponíveis e às vezes grandes princípios e escrúpulos morais são ignorados por agentes do governo encarregados da luta contra o terrorismo. Este artigo analisa a operação de resgate de reféns "Chavin de Huantar", destinada a libertar 72 pessoas sequestradas pelo Movemento Revolucionario "Tupac Amaru" 
(MRTA). O objetivo do estudo é explorar o 'dilema' da complexa questão de saber se uma 'democrácia liberal' pode ser considerado aceitável 'combater o terror com violência sem limites', e se nesta época de terrorismo, podemos resistir à tentação de 'não tem piedade'. Como metodologia, uma abrangente revisão da literatura e estudo de campo cujos resultados permitem verificar que não pode conceder concessões a terroristas nos reféns é feito, mas se deve priorizar o uso dos recursos mais adequados para os cidadãos vítimas deste flagelo sair ileso desta condição.

Palavras-chave: Contraterrorismo, Operações Especiais, Resgate de Reféns, Terrorismo.

\section{INTRODUCCIÓN}

Si se contempla el mundo que nos rodea, queda claro que las expectativas de un mundo mejor, surgidas al término de la Guerra Fría, eran infundadas y en este orden de ideas, resalta la irrupción de nuevas formas de amenaza a la seguridad internacional, entre las cuales sobresale el secuestro y la toma de rehenes, como un fenómeno terrorista que afecta directa e indirectamente a muchos países, y cuya faceta perturbadora refleja la cambiante relación existente entre la política y la guerra.

Para Ignatieff, la situación se torna más compleja "si asociamos la toma de rehenes con la llamada era de la guerra contra el terrorismo global, donde para derrotar el terror se requiere violencia, y en muchas ocasiones también se puede requerir coacción, engaño, secreto y violación de derechos" (2004, p.7).

En una democracia liberal, el uso de la fuerza coercitiva en contra del terrorismo, muchas veces puede ser considerado un 'mal menor' tornando difícil actuar ante una emergencia terrorista manteniendo un respeto absoluto en pro de los derechos humanos.

El terrorismo ha logrado deformar el desarrollo institucional de las democracias y con ello ha sido inevitable que se desate la violencia. Una violencia, que según Ignatieff, "se presenta en la lucha entre un Estado constitucional liberal y un enemigo terrorista, cayendo muchas veces en el más puro nihilismo, es decir, en la violencia por la violencia" (2004, p.11).

Para combatir las acciones terroristas, los gobiernos actúan haciendo uso de todas sus capacidades y en muchas ocasiones 'los grandes principios' y 'los escrúpulos morales' pierden su influjo sobre los agentes del Estado encargados de la lucha contraterrorista.

Los agentes estatales actúan guiados por ideales muy altos pero a veces traicionan sus creencias iniciales bajo la justificación de que aquello constituye un 'mal menor' ante el 'mal mayor' que representa el terrorismo. 
El presente artículo examina la actuación de los agentes del Estado de Perú, quienes el 22 de abril de 1997 ejecutaron una operación de rescate de rehenes destinada a liberar a 72 personas retenidas al interior de la casa del embajador de Japón en Lima, por parte de un grupo de terroristas del Movimiento Revolucionario Tupac Amaru (MRTA). También se analiza la actuación del MRTA, que no puede eludir su responsabilidad al utilizar el secuestro y la toma de rehenes como una herramienta terrorista y criminal para lograr sus fines.

A pesar de que en este caso se pudo liberar a los secuestrados, y se demostró la decisión de un gobierno legítimo para combatir el flagelo de la amenaza terrorista, se mantiene abierta hasta hoy una polémica referida a los pormenores de dicha operación; toda vez que entre otros aspectos, las propias investigaciones de la Comisión de Verdad y Reconciliación de Perú $(\mathrm{CVR})^{1}$, apuntan hacia una supuesta "eliminación metódica y programada" de algunos de los emerretistas.

En su Informe Final (CVR, 2003, p.719), la citada Comisión señala que:

La Operación de Rescate de los rehenes de la embajada japonesa fue una acción valerosa de las Fuerzas Armadas cuyos integrantes arriesgaron sus vidas y cumplieron su deber al enfrentar exitosamente una situación compleja para el país. Además de este reconocimiento explícito a las Fuerzas Armadas, la CVR admite también que existen suficientes elementos para presumir razonablemente que durante el operativo de rescate se habrían incurrido en actos violatorios a los derechos humanos. Por eso resulta imprescindible una investigación, con imparcialidad e independencia, a fin de determinar las responsabilidades del caso.

Con este estudio, el Autor pretende adentrarse en el complejo 'dilema' referido a si en una 'democracia liberal' se puede considerar aceptable 'combatir el terror con una violencia sin límites' y si en esta época de terrorismo, es posible resistirse a la tentación de 'no tener piedad'. Desde ya se piensa que la tarea no es simple, toda vez que no existe mayor reto político para la sociedad actual que intentar ganar la guerra contra el terrorismo sin perder el espíritu democrático.

1. La Comisión de la Verdad fue creada el 04 JUN 2001, mediante D. S. No 065-2001-PCM, siendo ratificada y complementada el 04 SEP 2001, bajo el nombre de Comisión de la Verdad y Reconciliación, siendo establecida por D.S. N ${ }^{\circ} 101-2001-P C M$. 


\section{LA OPERACIÓN DE RESCATE DE REHENES “CHAVÍN DE HUANTAR"}

\section{Situación del MRTA ${ }^{2}$}

El terrorismo en Perú, alcanzó su mayor connotación a inicios de la década de los 80, a través de dos organizaciones, Sendero Luminoso y el MRTA. Ambos impusieron una estrategia de terror que se prolongó durante 14 años, causando terror, desolación y muerte. Los ciudadanos peruanos vivieron en un verdadero campo de batalla, que abarcaba la ciudad y el campo, y en donde la economía nacional, la cultura, la libertad, los valores y la propia vida fueron prácticamente arrasados ${ }^{3}$.

El MRTA fue fundado en 1984 y su origen se remonta al partido Alianza Popular Revolucionaria Americana (APRA) rebelde y al Partido Comunista, los que luego de sucesivos fraccionamientos se fusionaron en el Movimiento de Izquierda Revolucionario El Militante (MIR-EM) y el Partido Socialista Marxista Leninista (PS-ML), conformando el Frente denominado La Convergencia que, en 1982, adoptó el nombre de Movimiento Revolucionario Túpac Amaru (MRTA) e inició su denominada Guerra Revolucionaria Popular el 22 de enero de 1984, con el ataque al puesto policial de Villa El Salvador, en Lima ${ }^{4}$.

Durante 1985, mientras gobernaba el partido APRA, el MRTA organizó cuadros guerrilleros en la selva del departamento del Cuzco, que fueron desarticulados por las fuerzas de orden a mediados de 1986. Luego de este revés, el MRTA trasladó su centro operativo a las zonas cocaleras de los departamentos de Junín, Huánuco y San Martín, formando frentes guerrilleros que le permitieron establecer una alianza con las mafias del tráfico ilícito de drogas.

El cabecilla inicial del MRTA fue Víctor Polay Campos, alias "Rolando", quién organizó los primeros actos violentos. Con su captura, en febrero de 1989, fue desarticulado el Comité Regional del Centro ubicado en los departamentos de Junín y Pasco. Dicha captura se produjo luego de un exitoso operativo en el que fueron abatidos otros 106 terroristas $^{5}$.

2. Para un análisis detallado de las acciones terroristas del MRTA, es recomendable confrontar dos textos fundamentales: 1) CVR, (2003); y 2) CPHE, (2010).

3. La cifra estimada más probable de víctimas fatales corresponde a 69.280 personas, una cantidad de pérdida de vidas humanas que supera ampliamente el número de víctimas sufridas por el Perú en todas las guerras externas y guerras civiles en 182 años de vida independiente. Del total de estas víctimas, el 79 \% corresponde a población rural y de ellas, el 56 \% se ocupaba en actividades agropecuarias (campesinos). Por otra parte, se ha podido determinar que el $75 \%$ de las víctimas fatales tenían el quechua u otras lenguas nativas como idioma materno.

4. El accionar terrorista del MRTA, según la CVR, dio como resultado más de 1.000 víctimas a lo largo de los años.

5. La captura de Víctor Polay y la muerte de los emerretistas fue atribuido a una iniciativa del ex Ministro de Defensa, General (R) Enrique López Albújar, motivo por lo cual el MRTA decidió planificar su asesinato, lo que se concretó en 1990. 
En el período de la denominada guerra revolucionaria del pueblo, el MRTA fue experimentando un paulatino crecimiento en su estructura política y militar ${ }^{6}$. De esta forma, a comienzos de la década del 90, el MRTA contaba ya con cuatro Frentes guerrilleros localizados en los departamentos de San Martín, Ucayali, Pasco, Junín, Cuzco y Puno. En cuanto a su actividad urbana, esta se centralizaba principalmente en las ciudades de Lima, Trujillo, Huancayo y Arequipa.

En julio de 1990, durante el gobierno de Alan García Pérez, Víctor Polay se fuga de la cárcel de Lima, junto a otros 46 militantes, utilizando un túnel construido probablemente con apoyo desde el interior de la misma cárcel. De esta forma, retoma la conducción del MRTA, apoyado esta vez por dos facciones legales de la izquierda radical, el Bloque Popular Revolucionario (BPR) y el Movimiento Patria Libre (MPL), convirtiéndose así en los principales instrumentos de captación y formación ideológica de cuadros emerretistas.

Con la llegada del régimen de Fujimori, a mediados de 1990, se observa un significativo cambio de actitud frente al terrorismo, marcándose un punto de ruptura dentro de la vertiginosa caída hacia una crisis generalizada del Perú.

Fujimori, se comprometió desde un primer momento a asumir la 'conducción directa' de la lucha contra el terrorismo, para lo cual definió una estrategia orientada a erradicarlo definitivamente.

La nueva estrategia se basaba en cuatro conceptos: 1) replanteaba el rol de las Fuerzas Armadas (FFAA), las cuales sin descuidar la misión específica señalada por la Constitución, debían volcar su acción, experiencia, infraestructura y vocación de servicio hacia el desarrollo de acción cívica y apoyo a la población, actuando militarmente sólo cuando fuese necesario enfrentar los grupos armados terroristas; 2) potenciaba significativamente el Sistema de Inteligencia Nacional

6. El crecimiento del MRTA puede ser visualizado a través de la escalada de sus acciones a lo largo del tiempo. De esta forma podemos observar que durante el año 1984 se registraron 19 actos terroristas; al año siguiente se produjeron 198 acciones de este tipo; el año 1986 llevaran a cabo 219 actos; en 1987 se efectuaron 392 actos; en 1988 llegaron a 413 actos y en 1989 cometieron 580 atentados, una cantidad 30 veces superior a las cometidas en sus inicios.

7. Según la CVR, las fuerzas militares actuaran ejerciendo una 'represión' indiscriminada contra la población 'sospechosa' de pertenecer o apoyar al PCP-SL o al MRTA. Posteriormente, esta estrategia se habría tornado más 'selectiva', aunque continuó posibilitando numerosas violaciones de derechos humanos. Esta práctica de excesos, de acuerdo con la opinión de la CVR, no solamente se identificó con actuaciones 'individuales' de oficiales o tropa, sino que también se relacionó con prácticas 'generalizadas' o 'sistemáticas' de violaciones de los derechos humanos, que constituyen en muchos casos crímenes de lesa humanidad así como trasgresiones de normas del DIH. Lo anterior es absolutamente rebatido en las entrevistas realizadas por el Autor a distintas autoridades militares en servicio activo y en retiro. Según dichas fuentes, no se puede hablar de prácticas generalizadas ni sistemáticas, por cuanto no existen antecedentes documentales tales como doctrinas, reglamentos, planes, procedimientos $u$ órdenes escritas donde se encuentren 'dispuestas' tales trasgresiones a los derechos humanos. En este mismo contexto, se reconoce la existencia de determinados crímenes pero son asociados con responsabilidades de orden individual, las cuales además podrían corresponder a reacciones psicológicas poco estudiadas por la CVR, tales como el "estrés de combate". 
(SIN), dotándolo de profesionalización y tecnología adecuada, para que así pudiese actuar coordinadamente y contase con los órganos operativos necesarios para orientar el esfuerzo de búsqueda hacia la ubicación y captura de los principales líderes terroristas ${ }^{8}$; 3 ) creaba los mecanismos institucionales, destinados a canalizar la participación activa, espontánea y democrática de la población, con el propósito de aislar a los grupos terroristas y demostrar que carecían de todo respaldo de la población ${ }^{9}$; y 4) reestructuraba el Poder Judicial, dotándolo de legislación adecuada para la defensa del Estado Democrático ${ }^{10}$.

Se calcula que en 1991, el MRTA tenía una fuerza que no superaba los 1.000 hombres, de los cuales el $60 \%$ estaba armado, gracias a ganancias derivadas de secuestros, toma de rehenes y vínculos establecidos con los narcotraficantes. Durante ese mismo año, el MRTA se fortaleció militarmente al re-incorporar a militantes evadidos de la cárcel e incrementar las acciones violentas urbanas y rurales.

A partir de 1992, comienza la decadencia del MRTA, debido a la acción de las fuerzas de orden, las que entre otros éxitos, lograron recapturar a Víctor Polay Campos y a otros integrantes de alta jerarquía. Adicionalmente, en el seno del BPR y del MPL surgieron discrepancias y disputas por el mando, lo que debilitó su trabajo a nivel popular y provocó el distanciamiento de militantes desilusionados y, cuando se capturaron y encarcelaron a sus cabecillas, ambos núcleos se disolvieron.

8. Según la CVR, lo anterior acrecentó las graves violaciones a los derechos humanos perpetradas por diversos agentes estatales, y gracias a las amplias atribuciones concedidas por ley, el SIN empezó a planear y ejecutar operaciones especiales de inteligencia por cuenta propia, utilizando para ello personal de unidades operativas de las FFAA, como si fuera personal a su disposición.

9. La CVR es bastante crítica respecto a la 'participación' ciudadana en la lucha contraterrorista. En este contexto, señala que los llamados "comités de autodefensa" (CAD) se organizaron por la 'presión' y el 'amedrantamiento' de las FFAA y de otros actores, y que como consecuencia de aquello fueron más allá de tareas de autodefensa y resultaron responsables de numerosos crímenes.

10. Según la CVR, estas disposiciones endurecieron la legislación terrorista, pero no contemplaron el respeto de garantías mínimas del debido proceso. Por otra parte, también se promulgaron varios Decretos Ley para extender las 'prerrogativas' militares, ampliando su poder en las zonas de emergencia y en la actividad contrasubversiva. Por su parte, el Ejército discrepa con lo señalado por la CVR y considera que el nuevo marco legal vino a suplir las deficiencias de un aparato jurídico endeble, desprovisto de mejores armas y recursos para poner freno al terrorismo. En este sentido argumenta que la respuesta del gobierno, a través de la dictación de la Ley del Sistema de Defensa Nacional (Decreto Legislativo $N^{\circ}$ 743), permitió 'comprometer' oficialmente al Estado en el combate contra las organizaciones terroristas (como participante 'de hecho' y no meramente nominal). Por otra parte, señala que el Decreto Ley No. 25.475 permitió implantar la naturaleza de las penas para los delitos terroristas, estableciendo además los procedimientos para la investigación, la instrucción y el juicio correspondientes para estos casos. En cuanto al Decreto Ley No. 25.659, que estableció el "delito de traición a la Patria" opina que con ello se logró disuadir y castigar adecuadamente a quienes se comprometieran con este tipo de delitos. Finalmente, en este ámbito legislativo, el Ejército destaca que por medio de la dictación del Decreto Ley 25.499, se pudo configurar una necesaria "Ley de Arrepentimiento" que serviría luego para lograr 'desarticular' grandes cuadros terroristas. 
Capturado Víctor Polay, asumió como Jefe Militar y miembro del Comité Ejecutivo del MRTA, Néstor Cerpa Cartolini quién trató de reorganizar la golpeada estructura organizacional.

En 1993, la violencia del MRTA disminuyó ostensiblemente y sus actos fueron relegados hacia fines netamente propagandísticos. En 1994, por efecto de la "Ley de Arrepentimiento", aumentaron las 'deserciones' y 'colaboraciones' con las fuerzas de orden, aumentando así el desbande emerretista ${ }^{11}$.

En 1995, se pone en evidencia la falta de conducción y dirección del Comité Regional del Centro, lo cual desnuda la desorganización que existe al interior de la estructura militar y política del MRTA. Esto se agrava con la detención de veinte dirigentes jerárquicos, incluyendo a la propia mujer de Néstor Cerpa Cartolini, Nancy Gilvonio Conde. A partir de este momento, las acciones comienzan a declinar hasta llegar a tan sólo 44 eventos en 1996, lo que representa apenas un $12 \%$ del nivel obtenido a inicios de la década.

Referido a la participación del MRTA en los delitos de secuestro y toma de rehenes, la CVR destaca que entre los hechos ilícitos imputables al MRTA, la toma de rehenes y los secuestros con fines políticos o económicos tuvieron un impacto particular en la sociedad peruana, dadas la forma y las condiciones en que se llevaron a cabo:

Durante los años de 1984 a 1996, la CVR ha obtenido evidencias que le permiten concluir que el MRTA habría realizado decenas secuestros individuales y colectivos, con fines de extorsión. Sin duda, la tasa real de secuestros es bastante superior, puesto que esta afirmación se basa en denuncias e investigaciones de oficio realizadas por las fuerzas del orden. En muchos casos, los individuos afectados no denuncian este delito en la esperanza de liberar a la víctima más fácilmente si no se ponen en contacto con las autoridades o ante el temor de verse acusadas de colaborar con el terrorismo cuando deben dar algo a cambio de ver a sus familiares libres. En este periodo la práctica de los secuestros no se realizó de manera uniforme sino que presentó diferentes momentos en los que se produjeron incrementos y descensos significativos. (CVR, 2003, p.550)

En el periodo comprendido entre 1984 y 1987, el número de secuestros es bastante reducido pero significativo. Así, en noviembre de 1984, el MRTA inicia esta práctica con la captura del empresario José Onrubia Romero. En los años siguientes no se registraron casos de secuestros, hasta que en 1987 esta práctica recomenzó, disminuyendo nuevamente en 1988. A partir de 1989 el índice de secuestros empieza a incrementarse hasta 1992. Esto se

11. Según la CVR, las leyes de arrepentimiento establecidas por el gobierno, para 'obtener información' y 'capturar' los últimos reductos del MRTA, resultaron muy efectivas. Las Leyes 25.499 del 12 Mayo de 1992 y 26.220 del 13 de Agosto de 1993 de "Arrepentimiento sobre el delito de terrorismo", fueron aprobadas por Decreto Supremo № 015 93-JUS. 
vincula con la puesta en marcha de un plan político-militar aprobado en 1988, que consideró realizar diversas acciones a cargo de un equipo especializado del MRTA en este tipo de actos criminales (CVR, 2003).

En 1993, el número de secuestros disminuye a consecuencia de un operativo policial realizado en el mes de octubre, donde resulta detenido un numeroso grupo de miembros del equipo de secuestradores. Seriamente debilitado, este equipo no realizó acciones el año siguiente, sino que se dedicó a prepararse para reanudar sus actividades en 1995, cuando el número de secuestros se incrementa nuevamente. A fines de ese año, los emerretistas vuelven a recibir un fuerte golpe mediante un nuevo operativo. En dicha ocasión, se intervino una vivienda ubicada en el distrito de La Molina, Lima, donde fue apresado un número considerable de emerretistas y se incautó gran cantidad de armamento. Finalmente, en 1996 con la mayoría de los miembros y dirigentes detenidos en diversos penales del país, este grupo subversivo planifica la que sería su última acción, con la intención de liberar a sus compañeros (CVR, 2003).

Así, llegamos a diciembre de 1996, ocasión en que un grupo armado del MRTA, asalta la embajada de Japón en Lima, capturando a más de 600 rehenes pertenecientes a diversos sectores de la vida política y económica peruana, en lo que se considera fue la última 'gran acción' emerretista.

\section{Situación del objetivo (escenario)}

El escenario general en que se ubicaba la residencia del embajador japonés, y donde se llevó a cabo la operación de rescate se considera netamente urbano. El edificio había sido convertido en una fortaleza por el gobierno japonés ${ }^{12}$; era un inmueble antiguo, de paredes más sólidas de lo normal y con estructuras de protección adicionales, tales como un muro de 3,5 metros, rejas en todas las ventanas, vidrios a prueba de balas y puertas construidas o modificadas para soportar incluso el impacto de granadas.

Respecto de las condiciones defensivas que brindaba este inmueble, Bolívar, nos señala lo siguiente:

El inmueble constituía una gran ventaja para los terroristas por las facilidades que la residencia otorgaba para impedir, frustrar o desbaratar cualquier intento de las fuerzas del

12. Este inmueble ya había sido objeto de un atentado terrorista el 29 de diciembre de 1992, ocasión en que estalló un coche bomba cargado con dinamita, anfo y un bidón de gas frente a la sede diplomática. La explosión causó una veintena de heridos que circulaban por las inmediaciones. El atentado fue atribuido a Sendero Luminoso como consecuencia de un publicitado apoyo del gobierno japonés a las reformas económicas establecidas por Fujimori en dicha oportunidad. Derivado de aquel atentado, la sede diplomática fue sometida posteriormente a un 'fortalecimiento' y 'endurecimiento' de su infraestructura. 
orden por rescatar sanos y salvos a los rehenes. En dicho inmueble era mucho más fácil para los terroristas controlar físicamente a los 72 rehenes, torturarlos psicológicamente y a su vez tomar las medidas defensivas (bloqueo de acceso; minado de puertas, paredes y pasadizos; y edificación de obstáculos), para disuadir a las fuerzas del orden a realizar una incursión de rescate. (2004, p.1)

La captura de la embajada fue planificada durante ocho meses y, al momento del asalto, los emerretistas se encontraban fuertemente equipados. Su armamento no sólo les permitía soportar un largo asedio, sino que además podían incluso rechazar un hipotético ataque con vehículos de blindaje ligero. Poseían fusiles y pistolas automáticas de distintos tipos y calibres, granadas antipersonales, equipos de comunicaciones, máscaras antigases, e incluso armas de mayor potencia como lanza cohetes, minas antipersonales y explosivos destinados a la confección de trampas del tipo caza bobos ${ }^{13}$.

\section{Preparación y entrenamiento}

\section{Actividades previas}

Luego del asalto, Néstor Cerpa interpuso una serie de demandas que pueden ser resumidas así: exigencia de liberación inmediata de 465 miembros MRTA desde distintas prisiones en todo el país, incluyendo a su propia esposa; planteamiento respecto de la necesidad de realizar una revisión de las reformas gubernamentales neoliberales de libre mercado establecidas por el gobierno peruano; cuestionamiento al programa de asistencia extranjera de Japón en Perú, bajo el argumento de que esta ayuda beneficiaba solo a un estrecho segmento de la sociedad, y protesta en contra de lo que denuncia como condiciones crueles e inhumanas existentes en las cárceles peruanas ${ }^{14}$. También anunció que liberaría gradualmente a los rehenes que no estuvieran conectados con el gobierno peruano.

Fujimori hizo su primer anuncio oficial sobre la toma de rehenes, en un discurso televisado donde condenó los hechos y rechazó las demandas. En esa declaración no excluyó un intento de rescate armado, pero dijo que estaba dispuesto a explorar una solución pacífica a la situación.

En días posteriores, el Comité Internacional de la Cruz Roja (CICR) en Perú, actuó como intermediario entre el gobierno y los terroristas. De esta forma, en los meses siguientes fueron

13. El propio MRTA difundió un video que detallaba cómo se había planeado el asalto, recurriendo a maquetas y a una falsa ambulancia, donde se ocultó el grupo terrorista para alcanzar las inmediaciones de la residencia, y luego neutralizar a un vigilante, e introducirse en una casa aledaña a la embajada (El Comercio, 1997).

14. Este petitorio original del MRTA, a la postre quedó circunscrito única y básicamente a una intención de 'negociación' que permitiera el intercambio de los rehenes por los emerretistas encarcelados. Las otras peticiones dejaron de ser mencionadas por el MRTA durante el resto de la crisis. 
liberados gran parte de los rehenes; primero todas las mujeres y, luego otros rehenes, con excepción de 72 de ellos ${ }^{15}$.

Aparentando la búsqueda de una solución pacífica, Fujimori nombró un equipo para sostener conversaciones con el MRTA, incluyendo al embajador canadiense, Anthony Vicent, quién también había sido brevemente rehén, el arzobispo Juan Luis Cipriani y un funcionario del CICR. Fujimori incluso habló con Fidel Castro, levantando especulación mediática sobre un eventual 'acuerdo' para que los emerretistas se asilaran en Cuba.

Prácticamente al mismo tiempo que se iniciaron las conversaciones negociadoras, el presidente Fujimori ordenó planificar una opción militar, y para tal efecto, fue conformada una 'Fuerza de Intervención', cuyos efectivos debían poseer entrenamiento de fuerzas especiales (comandos) y experiencia en combate. De este modo, fueron convocados integrantes de organizaciones de élite, tales como la División de Fuerzas Especiales del Ejército (DIFE) y las Fuerzas de Operaciones Especiales de la Marina de Guerra (FOES), los cuales se prepararon minuciosamente para enfrentar a los terroristas.

A inicios de febrero de 1997, tropas peruanas fuertemente equipadas asumieron la vigilancia de la embajada, y procedieron a reproducir música militar a alto volumen y a hacer gestos provocativos a los terroristas, los cuales respondieron con una ráfaga de disparos.

Al respecto, Kimura, expresa lo siguiente:

Este incidente, ocasionó que el Primer Ministro de Japón, recomendara públicamente al Perú a refrenarse de tomar riesgos innecesarios que podían hacer peligrar la vida de los rehenes. De esta forma, Fujimori fue presionado para alcanzar algún tipo de acuerdo negociado con los rebeldes del MRTA y asegurar así una liberación segura de los rehenes. Posteriormente, Fujimori se reunió con Hashimoto en Canadá, tras lo cual ambos líderes anunciaron que estaban de acuerdo sobre cómo manejar la situación de los rehenes, pero proveyeron de pocos detalles. $(2005$, pp.135, 136).

El 10 de febrero de 1997, Fujimori viajó a Londres, donde anunció que buscaba un país que diera asilo a los emerretistas, declaración que sorprendió a observadores y periodistas, ya que contradecía su posición inicial, previamente manifestada, en el sentido de no negociar con una organización terrorista.

15. En relación a los motivos para la liberación de este gran número de rehenes, más que los buenos oficios de la CICR aquello se debió a que era prácticamente imposible mantener retenida al interior de un inmueble de estas características a una cantidad superior a unas 50 personas. 
Lo que sí era efectivo, es que al mismo tiempo se había comenzado a construir bajo la residencia del embajador japonés, una compleja red de túneles, con diferentes accesos y pasillos subterráneos para así poder acceder a la residencia de la embajada; mientras se trabajaba en estos túneles, los planificadores de la operación de rescate, comentaron la similitud que guardaban estos, con las ruinas de unos templos pertenecientes a la ancestral cultura peruana pre-inca, y se determinó asignar a la operación el nombre clave que designa esas ruinas, "Chavín de Huantar"16.

\section{Actividades de inteligencia}

Durante la crisis, que se prolongó hasta abril de 1997, el gobierno no podía arriesgarse a efectuar un movimiento militar que pudiera poner en riesgo la vida de los secuestrados, debido a las consecuencias nacionales e internacionales que aquello ocasionaría. En consecuencia, se determinó realizar una 'maniobra de engaño' consistente en demostrar una supuesta apertura para negociar, mientras se preparaba la 'opción militar', medida que se considera muy semejante a lo sucedido durante la crisis de rehenes israelitas secuestrados por terroristas palestinos en el aeropuerto de Entebbe en Uganda ${ }^{17}$.

De esta forma, durante 126 días, el gobierno peruano simuló negociar con los terroristas, al mismo tiempo que entrenaba a sus fuerzas especiales en una réplica de la residencia. Durante las mañanas, se emitían marchas militares con megáfonos en los alrededores de la casa, respecto de lo cual, los medios de comunicación especularon que se trataba de una maniobra destinada a afectar la moral de los terroristas. El verdadero motivo de estas actividades era silenciar las maniobras destinadas a evacuar la tierra extraída durante la noche, por medio de camiones que salían desde una casa ubicada en la calle posterior de la residencia.

Como parte de las necesarias actividades de inteligencia, en preparación para el asalto, uno de los rehenes, fue provisto clandestinamente de una radio miniatura y se le dieron instrucciones cifradas para que advirtiera al resto, diez minutos antes de que comenzara la operación militar y les dijera que se mantuvieran lo más lejos posible de los emerretistas. Otra instrucción transmitida a los rehenes, consistía en que durante el asalto planificado, vistiesen ropas de colores claros, para así distinguirlos fácilmente de los terroristas, que vestían habitualmente ropas oscuras ${ }^{18}$.

16. Para la construcción de los túneles de acceso a la residencia de la embajada, fueron empleados 60 mineros de la Sierra Central, los cuales organizados en tres turnos, trabajaron en las excavaciones durante las 24 horas del día, a lo largo de tres meses.

17. Durante la crisis de rehenes en Entebbe, Uganda, en el año 1976, las autoridades israelitas expresaron en forma explícita su intención de negociar una salida pacífica con los terroristas palestinos, pero sin embargo al mismo tiempo se puso en funcionamiento la planificación y ejecución de la operación de rescate "Thunder".

18. El rehén que apoyó todas las actividades de inteligencia desde el interior de la residencia de la embajada fue el almirante retirado de la Marina de Guerra del Perú Luis Giampietri, quién más tarde fuera elegido Vicepresidente del Perú durante el 2º gobierno de Alan García. Al momento del secuestro Giampietri era un veterano y experimentado especialista en operaciones de inteligencia y de fuerzas especiales. 
Otro aspecto, considerado clave para la evacuación de los rehenes, era la necesidad de poder concentrarlos en un solo lugar; esto fue facilitado involuntariamente por el propio líder terrorista, quién al escuchar ruidos que le hicieron sospechar que un túnel pudiese estar siendo cavado bajo el primer piso, ordenó a todos los rehenes ubicarse en el segundo piso y de esta manera facilitó lo requerido por las fuerzas especiales.

Previo a la acción, se logró instalar una apreciable cantidad de sofisticados micrófonos y cámaras de video en miniatura al interior de la residencia, los cuales fueron escondidos en libros, botellas de agua, juegos de mesa y en otros elementos solicitados por los rehenes, que fueron entregados por las organizaciones de derechos humanos y el CICR, sin saber de su existencia. El almirante Giampietri y otros oficiales militares rehenes, asumieron la responsabilidad de colocar estos dispositivos en lugares seguros al interior de la casa.

Desde el exterior, agentes de inteligencia efectuaron vigilancia con cámaras de TV y otros sistemas de escucha, y de esta forma, pudieron identificar movimientos y sonidos, tales como voces y ruidos metálicos, corroborando lo que ocurría al interior de la residencia.

Gracias a estos dispositivos electrónicos, se pudo determinar que pese a haber organizado su seguridad de manera cuidadosa y a que estaban particularmente alertas durante las noches, cada tarde a lo menos ocho de los emerretistas se dedicaban a jugar fútbol, en el interior de la residencia por espacio de una hora aproximadamente.

\section{Actividades de planificación}

Las características del inmueble, facilitaba la organización de la defensa de los terroristas, en tanto que para los comandos aquello constituía un sinnúmero de obstáculos, algunos conocidos y otros desconocidos. Derivado de lo anterior, las fuerzas especiales determinaron la necesidad de utilizar técnicas del tipo close-quarter combat (CQC), también conocidas como técnicas de "combate cercano" o de "combate en recintos cerrados"19.

Estas técnicas consideran acciones de desplazamiento, ubicación de blancos y disparos, que son utilizadas cuando los enfrentamientos se producen a distancias muy cortas (entre 2 a 3 metros o inferiores). Por medio de estas técnicas, los comandos peruanos podían recorrer pasadizos, atravesar puertas para ingresar a habitaciones, eliminar los obstáculos, y rescatar sanos y salvos a los rehenes.

19. Las técnicas CQC habían sido desarrolladas al interior de las fuerzas especiales peruanas desde hacía varios años atrás, mediante un entrenamiento que inicialmente fue otorgado por especialistas israelitas, lo que luego originó una 'escuela' propia derivada de la experiencia en la lucha contraterrorista. Mayores detalles de estas capacidades pueden ser revisadas en el texto de la CPHE (2010). 
Para evitar daños colaterales (sobre todo derivados de eventuales rebotes de proyectiles), las fuerzas especiales determinaron emplear armamento de calibre no superior a $9 \mathrm{~mm}$, y como técnica de disparo, se consideró el "tiro instintivo selectivo" (TIS). En relación a esta modalidad de disparo, el general EP José Williams Zapata ${ }^{20}$, señaló a la CVR que: "dicha técnica consiste en efectuar tres disparos en dos segundos a las partes vitales del adversario (cabeza y tronco) y posteriormente efectuar un tiro de seguridad en la cabeza del enemigo para asegurarse que quede totalmente fuera de combate" (2003, p.274).

Para acceder a la residencia, se planificó una combinación de métodos de ingreso desde distintos lugares, los cuales se encontraban asociados al uso de cargas explosivas. Se cavaron tres túneles; dos de ellos, daban al jardín exterior de la casa y un tercero, al interior de la misma (lo que permitía acceder a la sala, un lugar que los secuestradores habían despejado para realizar misas y para jugar fútbol). Los tres túneles permitían un ingreso sorpresivo y simultáneo de los comandos, los cuales previo a ello podían disponer de un completo panorama de la situación al interior de la residencia, gracias a los micrófonos infiltrados a través de los ductos existentes en el edificio de la embajada ${ }^{21}$.

Adicionalmente, se consideró la detonación de tres cargas explosivas simultáneas, instaladas en tres habitaciones diferentes del primer piso; una de estas cargas, debía ser ubicada al medio de la habitación donde habitualmente los terroristas se concentraban para jugar fútbol, con la idea de permitir tanto el ingreso de las fuerzas especiales como también eliminar el máximo de adversarios presentes en dicho lugar. A través de estos orificios, creados por las cargas explosivas, debían ingresar 30 comandos al interior del edificio, y eliminar a los terroristas sobrevivientes del MRTA, para así impedir cualquier intento destinado a alcanzar el segundo piso para asesinar a los rehenes.

Adicionalmente, se consideró realizar otros dos movimientos simultáneos: un asalto directo por la puerta de entrada, con 20 comandos, para unirse al resto en el interior de la sala de espera, donde estaba ubicada la escalera hacia al segundo piso; y emerger desde dos túneles ubicados en el jardín trasero de la residencia, y desde allí, ascender por escaleras hacia el segundo piso de la embajada, hacer volar una puerta a prueba de granadas del segundo piso y evacuar los rehenes, haciendo dos aberturas en el techo para poder eliminar a los emerretistas del segundo piso, antes que tuvieran tiempo para ejecutar a los rehenes.

20. El general José Williams Zapata, con el grado de coronel tuvo la responsabilidad de diseñar el plan de operaciones "Nipón 96" y asumió el mando de los 140 comandos que conformaron la "Fuerza de Intervención" en el asalto a la residencia del embajador de Japón en Lima.

21. Para un detalle pormenorizado de las actividades de planificación, entrenamiento y ejecución de la operación de rescate léase el texto de la CPHE (2010). 
Resulta necesario reiterar que el cumplimiento de la misión dependía fundamentalmente de la 'eliminación' o 'neutralización total' de los terroristas, para así impedir su reacción, que podía poner en peligro la vida de los rehenes al interior de la embajada, y por lo tanto, todas las medidas asumidas y planificadas, pueden ser resumidas en lo que expresa Bolívar respecto de las acciones de rescate con participación de fuerzas especiales:

La moraleja es que los efectivos Fuerzas Especiales tienen que asegurarse una y otra vez, que el obstáculo (el terrorista) ha dejado de ser un peligro actual o potencial. No es por falta de humanidad ni violación a los Derechos Humanos o a las leyes de la guerra, sino como dijo el prestigioso periodista Gustavo Gorriti, tan sólo un frío "imperativo operacional" para cumplir el objetivo encomendado, que en el caso de Chavín de Huantar, era el de rescatar sanos y salvos a los 72 rehenes. $(2004$, p.3)

\section{Actividades de entrenamiento}

En relación al entrenamiento, resulta pertinente considerar lo que señala el general EP Nicolás Hermoza $^{22}$ :

la concepción de esta etapa tuvo una atención prioritaria. En esta etapa, se realizaron todos los ensayos de la operación; ... Ilegándose incluso a efectuar una preparación y un entrenamiento en un inmueble real e idéntico a la residencia de la embajada del Japón, que fue construido por el Servicio de Inteligencia y en donde se desarrollaron las prácticas de técnicas de dominación de inmuebles con tiro real, y técnica de tiro instintivo selectivo (TIS) con tiro real diurno y nocturno. (Hermoza, 1997, p.136)

De acuerdo con lo anterior, todas estas técnicas fueron ensayadas y practicadas por los efectivos de fuerzas especiales, con el objeto de reducir al máximo la posibilidad de reacción de los terroristas, y poder así, evacuar lo más pronto posible a los rehenes; la idea de maniobra, era realizar una acción rápida, y reforzada por el factor sorpresa.

El 16 de abril de 1997, se realizó el ensayo final, bajo la supervisión directa del propio Fujimori, en su condición de Jefe Supremo de las Fuerzas Armadas, siendo acompañado por el general

22. El general Nicolás Hermoza Ríos se desempeñó como Comandante General del Ejército durante el año 1991, y como Presidente del Comando Conjunto de las Fuerzas Armadas y Jefe del Comando Operativo del Frente Interno desde el año 1992 a 1998. Durante gran parte de su trayectoria profesional estuvo involucrado en la lucha contra el terrorismo y el narcoterrorismo en el Perú. En la actualidad cumple una condena de 8 años de prisión por estafa y apropiación indebida de dineros fiscales del Ejército y otra de 20 años por homicidio calificado, secuestro y desaparición forzada en distintos casos. Fue acusado de homicio calificado con ocasión de las ejecuciones extrajudiciales producidas durante la operación "Chavín de Huantar" pero luego resultó absuelto en este último caso. 
Nicolás Hermoza, responsable de la estrategia militar y por Vladimiro Montesinos Torres, responsable de la inteligencia requerida para la acción de rescate ${ }^{23}$.

\section{EJECUCIÓN Y RESULTADOS}

\section{Ejecución de la operación}

El 22 de abril de 1997, después de varios fracasos en la negociación y ante la perspectiva de que por este motivo, los secuestradores empezaran a negar la atención médica a los rehenes, Fujimori tomó la decisión de lanzar la operación de rescate.

Pasadas las tres de la tarde, el Presidente dio la orden de iniciar el operativo de rescate cuando Vladimiro Montesinos le informó telefónicamente que Néstor Cerpa Cartolini, el Árabe, Tito, Salvador, Cone, el Mexicano y los otros terroristas habían empezado su habitual partido de fútbol (Hermoza, 1997).

A las 14:30 hrs., los 140 efectivos de Fuerzas Especiales tomaron posición en los túneles y casas aledañas, esperando las órdenes definitivas. Cuarenta y siete minutos después, Fujimori, a través de un teléfono celular autoriza el inicio de la Operación. A las 15:23 hrs., tres detonaciones subterráneas hacen volar el piso de la sala, de la cocina, y del comedor, donde un grupo de terroristas jugaban fútbol. Las Fuerzas Especiales ingresaron por tres túneles que comenzaban en viviendas aledañas ... A las 15:23:10 hrs., una veintena de francotiradores se distribuyeron en techos de casas y edificios vecinos ... A las 15:23:25 hrs., otras agrupaciones de Fuerzas Especiales emergieron de las cuatro bocas del túnel principal y de otras dos, más pequeños, situados en el sector trasero del jardín ... Luego, el tiroteo se tornó intenso ... A las 15:23:32 hrs., salió el primer grupo de cuatro rehenes ... A las 15:29 hrs., salió un segundo grupo de cinco rehenes. A las 15:33 hrs., en medio del enfrentamiento, veintinueve rehenes alcanzaron la terraza de la residencia y se pusieron a cubierto. A las 15:35 hrs., un grupo de rehenes japoneses fueron evacuados hacia un túnel que desembocaba en una casa vecina. A las 15:37 hrs., otro grupo de rehenes japoneses se descolgaron por una ventana y alcanzaron otro túnel que desembocaba en una casa vecina ... A las 16:58 hrs., se ordenó el alto al fuego; las Fuerzas Especiales ocuparon totalmente el objetivo. (El Comercio, 1997, pp.210-211)

23. La participación de todas estas autoridades en la actividad de ensayo final originó que posteriormente, fuesen acusados de conformar una "cadena de mando paralela" para que un escuadrón clandestino de efectivos policiales y de inteligencia llevasen a cabo acciones de 'repaso' de heridos y ejecuciones 'extrajudiciales' durante la operación de rescate. 
La operación se puso en ejecución de acuerdo con lo planificado, entrenado y ensayado. La operación se ejecutó sin mayores dificultades hasta la orden de abrir los accesos de los túneles en el punto final. En ese momento se produjeron imprevistos que fueron superados por soluciones alternas. En el interior de la residencia, los terroristas respondieron con fuego cruzado de ráfagas cortas y largas; en algunos casos, los terroristas dispararon sin control una gran cantidad de munición y arrojaron granadas. Las Fuerzas Especiales, de acuerdo a lo ensayado, respondieron con tiros controlados y del tipo "tiro a tiro", empleando armas con silenciador y con gran disciplina de fuego, lo que permitió hacer en varias oportunidades tiro cruzado en condiciones seguras; en el primer piso, las Fuerzas Especiales se enfrentaron con tres terroristas y en el segundo, con seis; durante las acciones, 25 efectivos de las Fuerzas Especiales resultaron heridos. (Hermoza, 1997, pp.139-140)

La mayoría de los heridos de las fuerzas especiales, se produjeron en el corredor del segundo piso, en circunstancias que fueron atacados con granadas de mano arrojadas por los terroristas. Estas mismas explosiones, ocasionaron la activación de trampas instaladas en el lugar, las cuales deflagraron por simpatía.

Durante la acción, fueron muertos dos efectivos de las fuerzas especiales y resultó herido un rehén, quién falleció posteriormente de un paro cardíaco. Los comandos fallecidos fueron el comandante EP Juan Valer Sandoval y el teniente EP Raúl Jiménez Chávez. El rehén Carlos Giusti, fue herido en una pierna y sufrió una violenta hemorragia, que finalmente le provocó un paro cardíaco y la muerte, constituyéndose en el único rehén muerto durante la acción.

Respecto de las circunstancias en que resultaron fallecidos los efectivos de fuerzas especiales, se pudo determinar posteriormente que el comandante Valer, cayó abatido por un terrorista, en circunstancias que protegía la salida del canciller Tudela, hacia una terraza exterior de la residencia. Por su parte, el teniente Jiménez, resultó muerto en circunstancias que ayudaba a un compañero herido, siendo alcanzado por una granada arrojada por uno de los emerretistas.

En relación a los 14 terroristas muertos en el enfrentamiento, las versiones oficiales de la fuerza de asalto indican que los primeros fallecidos se produjeron a partir del amplio canal subterráneo desde donde se lanzó el ataque principal, que se inició con la apertura de tres huecos en el primer piso de la residencia. En este primer enfrentamiento habrían muerto 6 terroristas, incluido el propio líder del MRTA, Nestor Cerpa Cartolini; todos ellos habrían resultado sorprendidos por las fuerzas especiales, cuando subían las escaleras hacia el segundo piso. Otros 3 terroristas, habrían fallecido al enfrentarse con otro grupo de fuerzas especiales que se introdujo por una terraza en el ala derecha del segundo piso de la mansión, y finalmente, 5 terroristas habrían sucumbido como consecuencia de la explosión provocada por los militares en la sala mientras jugaban fútbol.

Dado que más de 140 efectivos irrumpieron en la residencia por distintos accesos y en forma simultánea, al enfrentar sus blancos, dispararon con gran precisión y como resultado 
de ello, todos los terroristas fueron abatidos. La distancia de enfrentamiento resultó ser inferior a los 3 metros, y por lo tanto, los impactos de los proyectiles produjeron gran daño en los cuerpos de los terroristas, y muchos de ellos presentaron una gran cantidad de proyectiles alojados en sus cuerpos, debido al empleo de las técnicas de tiro instintivo selectivo (TIS). Posteriormente, la utilización de esta táctica de Tiro Instintivo Selectivo (TIS) como método de neutralización de los terroristas, vendría a ser seriamente cuestionada, ya que sería interpretada como parte de un modo de operación destinado a 'rematar' a cualquier sobreviviente del MRTA ${ }^{24}$.

\section{Resultados de la operación}

\section{Consecuencias de la acción militar}

El día 22 de abril de 1997, Fujimori, luego recorrer el interior de la residencia de la embajada ya recuperada, felicitó a los comandos que en menos de una hora habían culminado exitosamente la operación de rescate, y posteriormente acompañó a los rehenes liberados en dirección al Hospital Militar. Sin embargo, a las 18:00 hrs. del mismo día, regresó a la embajada, y dio una conferencia de prensa ante una multitud de periodistas, resaltando que la operación de rescate se había efectuado de acuerdo a lo planificado, y que el éxito se debió a las capacidades de las fuerzas especiales y al apoyo brindado por el Servicio de Inteligencia (SIN).

El tema de discusión posterior, se va a centrar en la muerte de todos los terroristas, que terminó siendo sindicada como una 'ejecución extrajudicial'. Si aquello se hubiese producido, sería algo totalmente objetable respecto de la planificación original, la cual de acuerdo a los antecedentes revisados, no consideraba en ningún término la disposición de dar de baja a los terroristas que fuesen capturados como consecuencia de su propia rendición ${ }^{25}$.

Sin embargo, posteriormente, incluso las muertes producidas durante el enfrentamiento, serían objetadas por la CVR en donde se señaló expresamente lo siguiente:

24. Respecto a estas técnicas de disparo, se puede mencionar que en mayo de 1980, durante una operación de rescate realizada por efectivos del Special Air Service (SAS) del Reino Unido de Gran Bretaña en la embajada Iraní en Londres, uno de los terroristas presentó 82 impactos de bala (Campbell, 1980).

25. Derivado de las investigaciones posteriores se ha podido determinar que luego de la acción de la Fuerza de Intervención a cargo del entonces coronel José William Zapata, habría ingresado a la residencia del embajador de Japón, un destacamento clandestino de 20 efectivos policiales y de inteligencia bajo las órdenes del teniente coronel Jesús Zamudio, con la misión específica de 'repasar' los cadáveres de los emerretistas y ultimar cualquier sobreviviente. Esa sería la razón por la cual varios cadáveres, ya muertos en combate, aparecieron en las autopsias con perforaciones de balas en la nuca. Por lo tanto, este sería el personal que ejecutó al único sobreviviente, Eduardo Nicolás Cruz Sánchez, alias 'Tito'. 
La información obtenida durante la investigación del Ministerio Público permite afirmar que la orden de rematar a los subversivos formaba parte del modo de operación que se empleó durante las acciones de rescate de los rehenes. En este sentido resulta claramente ilustrativa la declaración del Teniente coronel EP Luis Alberto Donoso Volpe, integrante del Equipo Delta, Grupo 5, quién describió la técnica de Tiro Instintivo Selectivo empleada durante el operativo, según la cual se dispararon contra los subversivos un aproximado de dos a tres balas por comando y uno de los comandos se encargaba de verificar si se encontraba vivo o muerto el delincuente, y de acuerdo a la técnica se efectuaba el tiro de remate. El citado oficial precisó que de acuerdo a la técnica, si estaba con vida el delincuente se le daba el tiro de remate. (Ignatieff, 2004, p.76)

Como resultado de las muertes de los integrantes del MRTA, se generaron diversas denuncias, por parte de familiares de los terroristas fallecidos y de agrupaciones de derechos humanos, respecto de la supuesta existencia de ejecuciones extrajudiciales ${ }^{26}$. De hecho, según antecedentes posteriores, se llegó a afirmar que al menos 3 terroristas, entre ellos Eduardo Cruz Sánchez (alias Tito), se habrían rendido pero aparecieron muertos al finalizar la operación ${ }^{27}$.

En un contexto similar, la propia Agencia de Inteligencia de Defensa de los Estados Unidos de América (EE.UU.) (DIA), dio a conocer un informe desclasificado donde se señala que uno de los terroristas del MRTA, Ilamado Roli Rojas, el día de las acciones habría sido descubierto intentando huir de la residencia mezclado con los rehenes, y que un comando lo habría detenido, para luego llevarlo a la parte trasera de la casa y allí ejecutarlo con una ráfaga de ametralladora, que incluso le habría volado parcialmente la cabeza ${ }^{28}$.

Según el periodista David Hidalgo Vega, al ser desclasificados los "informes de necropsia" ${ }^{29}$ se pudo constatar que los cadáveres de los integrantes del MRTA presentaban una gran cantidad de

26. La exhumación de los cadáveres de los terroristas del MRTA, se realizó en Marzo de 2001, y estuvo a cargo del Equipo Peruano de antropología Forense EPAF. Ocho de los cuerpos examinados presentaban disparos en la cabeza desde atrás hacia delante, una dirección considerada como atípica en un enfrentamiento. Según este peritaje, en el momento en que se efectuaron los disparos, las víctimas habrían estado carentes de movilidad o en una condición de movilidad casi nula (CVR, 2003).

27. El general PNP Máximo Rivera, ex jefe de la DINCOTE, reveló haber 'escuchado' a un terrorista pidiendo perdón. Asimismo, el congresista Xavier Barrón, declaró que un amigo suyo, también ex rehén, había visto a otro terrorista rendirse. Los policías Raúl Robles Reynoso y Marcial Torres Arteaga, se convirtieron en testigos claves en estas presuntas ejecuciones extrajudiciales, ya que según sus declaraciones, tras culminar el operativo, capturaron vivo Eduardo Cruz Sánchez (alias Tito), y lo entregaron a un militar enviado por el Teniente Coronel EP Jesús Zamudio Arteaga.

28. Estos argumentos han quedado descartados ya que el emerretista que habría intentado huir mezclado entre los rehenes liberados sería Eduardo Cruz Sánchez alias "Tito" y no Roli Rojas. No obstante, esta información desclasificada de la DIA puede ser confrontada en Defense Intelligence Agency (1997).

29. Según el Informe de Necropsia No. 06, que describe David Hidalgo, Néstor Cerpa presentaba 39 impactos de bala; el Informe $\mathrm{N}^{\circ} 03$ indica que Roli Rojas presentaba 26 impactos; y el Informe $\mathrm{N}^{\circ} 14$ señala que Tito presentaba 2 impactos. 
orificios de bala, aspecto que se consideró como característico a este tipo de enfrentamiento. Sin embargo, al ser exhumados los restos de todos ellos, al menos ocho presentaron disparos a la misma altura en la parte trasera del cuello, lo cual sugiere que la distribución y recurrencia de estas lesiones las convierte en un patrón (Hidalgo, 2007).

Derivado de todo lo anterior, en mayo de 2001 se iniciaron las detenciones preliminares de los acusados por estas presuntas ejecuciones extrajudiciales, en circunstancias que al mismo tiempo, algunos congresistas planteaban pedidos de amnistía para todos los militares que habían participado en el operativo de rescate.

En junio de 2002, se inició el juicio a los comandos y luego, en agosto del mismo año, se trasladó al fuero militar el juzgamiento de los efectivos participantes en dicho operativo de rescate, bajo el argumento de que los hechos correspondían a un delito "de función", toda vez que la acción se habría producido en una "zona declarada en estado de emergencia".

El 12 de noviembre de 2003, la Sala de Guerra resolvió archivar la causa por el delito de abuso de autoridad y homicidio calificado seguida contra los efectivos de fuerzas especiales participantes en la operación "Chavín de Huántar", por no existir prueba alguna que acreditara su responsabilidad ante dichas acusaciones, y de esta forma, el 05 de abril de 2004, se confirmó la resolución que archivó la causa. Posteriormente, se abrieron otras causas en contra de distintos niveles de autoridades participantes en dicho operativo, pero se consideró que los diferentes cargos habían prescrito, a causa del tiempo trascurrido desde la fecha del rescate de los rehenes.

Hasta la fecha, esta situación no ha sido resuelta definitivamente, y de hecho con fecha 23 de febrero de 2012, la Corte Interamericana de San José de Costa Rica (Corte IDH) notificó al Estado de Perú acerca de la admisión de una demanda presentada por la Comisión Interamericana de Derechos Humanos $(\mathrm{CIDH})$ por las presuntas ejecuciones extrajudiciales producidas durante el operativo de rescate ${ }^{30}$.

\section{El objetivo de la misión: ¿valía la pena el riesgo?}

En la operación "Chavín de Huantar", los riesgos políticos fueron altísimos; los terroristas y secuestrados se encontraban en territorio japonés y uno de los rehenes era el propio embajador de

30. En relación a la 'ejecución extrajudicial' de Eduardo Cruz Sánchez alias "Tito", todas las evidencias apuntan a la participación del ya mencionado "destacamento clandestino". Estas evidencias son varias y concretas: 1) no formaban parte del contingente de comandos que llevó a cabo el rescate; 2) tampoco participaron en la planificación, coordinaciones ni ensayos del operativo; 3) se diferenciaban de los 140 comandos por el hecho de no usar cascos sino que pasamontañas negros que ocultaban sus rostros e ingresaron por uno de los túneles cuando todo había concluido; 5) al respecto, existen numerosos testimonios gráficos que acreditan este ingreso clandestino y la presencia de este personal, en las fotografías que fueron tomadas una vez concluida la operación. 
ese país ${ }^{31}$. Habían funcionarios diplomáticos y de empresas japonesas que también estaban cautivos. Entre los rehenes se encontraban otras altas autoridades, tales como el embajador boliviano, el canciller peruano, así como magistrados y funcionarios, tanto peruanos como extranjeros.

Por otra parte, la opinión pública no apoyaba totalmente al gobierno y a sus instituciones, en relación a la determinación de responsabilidades y a la buena o mala gestión efectuada posterior a la toma de rehenes. Incluso, el gobierno peruano recibió la presión adicional de a lo menos tres comunicados que fueron firmados por la mayoría de los rehenes retenidos al interior de la embajada, y en los cuales apelaban en forma pública para que las autoridades del país, se mostraran dispuestas a 'negociar' con el MRTA ${ }^{32}$.

Pese a todas estas presiones, desfavorables para la opción militar, el Presidente Fujimori consideró que este secuestro masivo era un acto terrorista, y que como tal, debía enfrentarlo de todas las formas posibles, y con todos los medios y recursos disponibles. Para tal efecto, y pese a que demostró una supuesta intención de negociación, mantuvo siempre vigente la opción de ejecutar una operación de rescate para liberar a los rehenes ${ }^{33}$.

En este contexto, se considera que su decisión es totalmente aceptable, y que el objetivo de rescatar a los rehenes, bien valía el riesgo de intentarlo ${ }^{34}$.

\section{¿Se desarrolló un plan destinado a alcanzar el éxito?}

Si bien es cierto, los efectivos de fuerzas especiales superaban en número a los terroristas y finalmente podían llegar a neutralizarlos, el problema radicaba en que mientras más tiempo durara el combate, era más probable que el desenlace afectara la seguridad de los rehenes, por lo tanto era necesario actuar en el más mínimo de tiempo.

31. Pese a estas restricciones, Fujimori ordenó realizar una acción militar de rescate, en forma unilateral y desoyendo las exigencias internacionales, especialmente las de Japón, en orden a no poner en riesgo la vida de los secuestrados. Se estima que el gobierno japonés se vio obligado a manifestar su beneplácito por la acción armada sólo con la intención de soslayar la responsabilidad del gobierno de Perú ante la Comunidad Internacional. Otro argumento podría estar radicado en el evidente rechazo de Japón a las acciones terroristas luego de la trágica experiencia vivida el año 1995, con ocasión del ataque con gas sarín ejecutado en el metro de Tokio por parte de integrantes de la secta Aum.

32. Posterior a su liberación, el propio canciller Tudela, declaró que dichos comunicados no debían ser tomados en serio, ya que habían sido escritos y firmados bajo coacción o en condiciones anormales. Para mayores antecedentes (El Comercio, 1997).

33. Tras un aparente intento del Presidente Fujimori de negociar la inmunidad y un salvoconducto para los secuestradores hacia países como la República Dominicana o Cuba, el 22 de abril de 1997, ciento veintiséis días después de que se iniciase la toma de rehenes, Fujimori ordenó efectuar la acción de rescate (N. del A.).

34. Pese a que durante la acción se produjeron bajas, se pudo alcanzar el objetivo de liberar a 71 secuestrados mediante una operación de "acción directa", y en medio de un ambiente de combate, donde necesariamente se debían producir muertos y heridos. 
De lo anterior, se puede deducir que para alcanzar la superioridad relativa, era necesario cumplir un 'imperativo operacional básico' consistente en 'eliminar' o 'neutralizar totalmente' al adversario, al inicio de las acciones, para así impedir su reacción, eliminando por completo todo riesgo de amenaza. Aquello implicaba que la eliminación de los adversarios fuese ejecutada en la forma más rápida posible, dado que cualquier demora en esta acción, aumentaba la posibilidad de que los terroristas atentaran contra la vida de los rehenes y de la fuerza atacante, sobre todo por el uso de armas de fuego, granadas de mano y artefactos explosivos en recintos cerrados, todo lo cual aumentaba enormemente tanto la posibilidad de daños directos como colaterales.

En consecuencia, se diseñó un plan que consistía en la inserción de un gran número de efectivos de las fuerzas especiales al interior de la embajada, los cuales debían ingresar en forma simultánea desde distintos lugares de acceso y haciendo uso de técnicas de CQC asociadas con técnicas de TIS, para así poder lograr el "efecto deseado".

\section{¿Hubo alguna circunstancia imprevista que afectara el resultado de la operación?}

En relación a las circunstancias imprevistas que afectaron a la operación, se puede destacar que la fase central de la operación de rescate estaba planificada con una duración de 16 minutos, sin embargo, la misma se duplicó en el tiempo, al durar 33 minutos. En este aspecto, la principal duda respecto de las acciones llevadas a cabo por los terroristas, radica en el antecedente de que pese a haber contado con el tiempo suficiente para reaccionar en contra de los rehenes, hayan optado por no sacrificarlos. Es en este mismo contexto, donde se ha creado una complicada polémica, en el sentido que según algunas opiniones, los terroristas no habrían tenido nunca la intención de asumir esta decisión extrema, sino que desde un principio habrían estado exclusivamente decididos a combatir, y por lo tanto, habrían resultado una parte de ellos muertos en los enfrentamientos y otros, habrían sido ajusticiados impunemente por las fuerzas de asalto.

Por otra parte, los mayores cuestionamientos a la operación, se producen posterior a la ejecución de la misma, luego que la televisión peruana mostrara al Presidente Fujimori caminando entre los rebeldes muertos, algunos de cuyos cuerpos estaban mutilados. En esas circunstancias, Fujimori fue fotografiado parado prácticamente sobre los cuerpos de Néstor Cerpa y Roli Rojas en la escalera principal de la residencia y de hecho, la cabeza destrozada de Rojas es perceptible en la fotografía. Inicialmente, esta victoria militar fue publicitada como un triunfo político y fue usado para reforzar la postura de línea dura de Fujimori, en contra de los grupos terroristas ${ }^{35}$.

35. De hecho, los índices de popularidad de Fujimori se duplicaron rápidamente hasta alcanzar cerca del 70\%, Ilegando a ser aclamado como un verdadero héroe nacional, ya que dentro de esta misma estrategia comunicacional, se llevó el crédito personal por la operación, al declarar en una entrevista en la edición de El Comercio del 17 de diciembre de 1997, que poco después que la residencia del embajador fuese tomada, había sido él quién había planeado personalmente la operación junto con el SIN comandado por Julio Salazar y Vladimiro Montesinos, y el Comando Conjunto de las Fuerzas Armadas bajo el mando del general de Ejército Nicolás Hermoza.

LA TOMA DE REHENES COMO ACTO TERRORISTA INTERNACIONAL. ANÁLISIS DE LA OPERACIÓN DE RESCATE "CHAVÍN DE HUANTAR" 
Cuando la operación finalizó, los cuerpos de los terroristas fueron retirados por los fiscales militares y no se permitió la entrada de representantes de la Oficina del Fiscal General. Los cadáveres no fueron llevados al Instituto de Medicina Forense para la autopsia requerida por ley, sino que las autopsias fueron realizadas en la morgue del hospital de la Policía. Los informes de las autopsias fueron mantenidos en secreto hasta el año 2001, y no se permitió que los familiares identificaran a sus deudos ni que conocieran los resultados de las autopsias. Los cuerpos fueron enterrados en secreto en cementerios a través de todo Lima.

\section{CONCLUSIÓN}

Según Marta Crenshaw (2006), la toma de rehenes puede ser analizada como una forma de negociación coercitiva, en la cual los rehenes "representan el poder para herir en su forma más pura, y desde esta perspectiva, los terroristas optan por tomar rehenes porque en situaciones de negociación la mayor fortaleza y disponibilidad de recursos del gobierno no cuentan en su ventaja" (p.76).

Esta forma de terrorismo constituye una forma de manipular las decisiones políticas de los gobiernos. La negociación terrorista es una forma de chantaje o extorsión, donde los terroristas toman rehenes con el objeto de influir en las opciones del gobierno.

La amenaza de muerte de los rehenes, se transforma en algo peor que el acatamiento de las demandas de los terroristas y por otra parte, en una situación de toma de rehenes los terroristas quedan atrapados junto a sus víctimas, lo cual torna aún más difícil la posibilidad de echarse para atrás, dado que el gobierno controla las rutas de escape.

En este contexto, se podría esperar una conducta desesperada por parte de los terroristas en caso de no ceder a sus demandas. Lo anterior, obliga al gobierno a efectuar un cálculo de los resultados asociados a esta compleja situación de crisis.

Respecto a la forma de liberar rehenes, la operación analizada presenta serios problemas y cuestionamientos, ya que luego de una espectacular operación militar los planificadores y ejecutores de dicha fueron cuestionados por graves violaciones a los derechos humanos, pues según los testimonios de algunos rehenes, la intervención militar se habría propuesto "evitar supervivientes" entre los autores del delito, llegando a disparar contra aquellos que se rendían, de tal manera que la acción finalmente se saldó con la muerte de los catorce secuestradores.

Al respecto, es necesario resaltar que la lucha contra el terrorismo no es una situación ajena al derecho, a pesar de que en el marco de este tipo de conflicto, sea frecuente que quienes toman parte directa en las hostilidades asuman conductas violentas, con amplio uso de una fuerza contundente y directa, pero la cual se exige deba ser comedida. 
En la toma de rehenes por parte de terroristas, las personas privadas de libertad afrontan las más dolorosas condiciones de existencia, pues no sólo se ven despojadas de su capacidad individual de autodeterminación, sino que padecen los rigores de una reclusión prolongada e inmisericorde.

Alejadas de sus familias y de sus entornos, los rehenes son sometidos bajo la voluntad omnipotente de sus captores, permaneciendo marginados de la comunicación con el exterior y expuestos a los rigores climáticos, al hacinamiento y a las enfermedades. Estas víctimas, sufren en carne propia la pérdida de todos los valores y el desconocimiento de todos sus derechos, y los efectos de estas privaciones, se proyectan más allá de la individualidad de las víctimas, golpeando moral, psicológica y económicamente a sus familiares.

Por lo tanto, ni el Estado, ni la sociedad civil, ni la comunidad internacional pueden permanecer indiferentes ante la injusta y lamentable suerte de estas personas. El problema radica en cómo el Estado puede lograr la liberación de estos rehenes, sin afectar su integridad física, sin producir daños colaterales y respetando los derechos humanos incluso de los criminales.

La experiencia derivada del análisis de este estudio de caso nos demuestra que ello no es simple, ya que por una parte, el Estado tiene la obligación de impedir que las personas sean sometidas a privaciones antijurídicas de la libertad, tales como la toma de rehenes. Por otra parte, el escrutinio público y la ética política en una democracia obligan a que los agentes estatales mantengan una fortaleza moral que les permita controlar 'su furia' hacia los terroristas.

A juicio del autor, la comunidad internacional se debe manifestar opuesta a realizar concesiones a los terroristas en materia de toma de rehenes, pero se debe priorizar la utilización de los recursos más apropiados para que los ciudadanos víctimas de este flagelo salgan ilesos de esta condición.

El terrorismo produce desorientación en una democracia liberal, ya que hace caso omiso de las virtudes que derivan de su libertad. Al respecto, tenemos que ser capaces de defendernos, con la fuerza de las armas, pero aún más con la fuerza del razonamiento, dado que las armas sin razones, se utilizan en vano.

El deber primordial de los líderes políticos en una democracia atacada por el terrorismo es mantener las fuerzas contraterroristas centradas con atención en los requisitos destinados a mantener la legitimidad. La única cura contra la violencia por la violencia (nihilismo), es que las sociedades democráticas insistan en que "el uso de la fuerza es legítimo" sólo hasta el punto en que "sirva a objetivos políticos defendibles".

Lo anterior, implica una estricta observación de las reglas de compromiso con respecto a la utilización de la fuerza mortífera y la prevención de daños colaterales. 
Finalmente, parece oportuno reflexionar respecto a lo que nos señala Eurípides en sus famosos monólogos: "Sé bien el mal que pienso hacer, pero más fuerte que todos mis remordimientos es mi furia, furia que a los mortales acarrea el mayor de los males" (2000, pp.111,112).

\section{REFERENCIAS}

Bolívar, A. (2004). Chavín de Huantar en el marco de la Teoría de Operaciones Especiales. En Vivir bien, 85.

Campbell, D. (1980). SAS Dares \& Wins: A raid that worked, Soldier of Fortune (pp. 26-29).

Comisión de Verdad y Reconciliación del Perú, CVR. (2003). Informe Final, Lima: Editorial CVR.

Comisión Permanente de Historia del Ejército, CPHE. (2010). En honor a la verdad, Lima: Editorial Gráficas Canepa.

Comisión Permanente de Historia del Ejército, CPHE. (2010). Operación Militar de Rescate de Rehenes Chavín de Huántar. Versión Oficial del Ejército del Perú, Lima: Editorial Gráficas Canepa.

Crenshaw, M. (2006). La lógica del terrorismo: el comportamiento terrorista como producto de una opción estratégica Terrorismo y contraterrorismo. Comprendiendo el nuevo contexto de la seguridad, Buenos Aires: Editorial ABRN.

Defense Intelligence Agency, DIA. (1997). Commando execution of two MRTA hostage takers and take no prisoners order, En Intelligence Information Report IIR, Estados Unidos de América.

El Comercio (1997). La crisis de los rehenes en el Perú. Base Tokio. Lima: Editorial El Comercio.

Eurípides (2000). Medea. En Tragedias I. Madrid: Editorial Gredos S.A.

Hermoza, N. (1997). Operación Chavín de Huantar. Rescate en la residencia de la Embajada del Japón. Lima: Editorial Fimart.

Hidalgo, D. (2007). Sombras de un rescate. Tras las huellas ocultas en la residencia del embajador japonés. Lima: Editorial Planeta Perú.

Ignatieff, M. (2004). El mal menor. Ética Política en una era de terror. Bogotá: Editorial Taurus S.A. 
Kimura, R. (2005). Alberto Fujimori. El presidente que se atrevió a soñar. México D.F.: Editorial Felou.

Ministerio de Justicia del Perú (1992-1993). Leyes de Arrepentimiento sobre el delito de terrorismo, Ley 25.499 del 12 Mayo de 1992 y Ley 26.220 del 13 de Agosto de 1993 aprobadas por Decreto Supremo № 015-93-JUS. Lima: Editorial del Ministerio de Justicia. 